\title{
Temporal distinguishability in Hong-Ou-Mandel interference for harnessing high-dimensional frequency entanglement
}

\author{
Yuanyuan Chen $\mathbb{D}^{1,2,3}$, Sebastian Ecker $\mathbb{D}^{1,2}$, Lixiang Chen $\mathbb{D}^{3 凶}$, Fabian Steinlechner $\mathbb{D}^{4,5 凶}$, Marcus Huber (D) $^{1}$ and Rupert Ursin $\mathbb{D}^{1,2 凶}$
}

High-dimensional quantum entanglement is currently one of the most prolific fields in quantum information processing due to its high information capacity and error resilience. A versatile method for harnessing high-dimensional entanglement has long been hailed as an absolute necessity in the exploration of quantum science and technologies. Here we exploit Hong-Ou-Mandel interference to manipulate discrete frequency entanglement in arbitrary-dimensional Hilbert space. The generation and characterization of two-, four- and six-dimensional frequency entangled qudits are theoretically and experimentally investigated, allowing for the estimation of entanglement dimensionality in the whole state space. Additionally, our strategy can be generalized to engineer higher-dimensional entanglement in other photonic degrees of freedom. Our results may provide a more comprehensive understanding of frequency shaping and interference phenomena, and pave the way to more complex highdimensional quantum information processing protocols.

npj Quantum Information (2021)7:167; https://doi.org/10.1038/s41534-021-00504-0

\section{INTRODUCTION}

Harnessing entanglement in high-dimensional systems may well play a central role in elevating the performance of advanced quantum information protocols towards practical applicability. In particular in the context of quantum communication, photon pairs entangled in high dimensions can carry more quantum information, making them compelling for enhancing quantum channel capacities, improving noise resilience, and even speeding up certain tasks in photonic quantum computation ${ }^{1-4}$. Several physical properties of photons can be used to directly encode high-dimensional entanglement, including orbital angular momentum ${ }^{5}$, time-energy ${ }^{6}$, and path $^{7}$. The employment of highdimensional encoding in the spatial domain has strict requirements on the quality of optical wave-fronts and shaping for generation and measurement ${ }^{8}$. In the past decades, elaborate fiber designs have enabled the transmission of high order spatial modes over a distance of several kilometers 9,10 , as well as some well-designed schemes are presented for realizing the highdimensional intracity quantum cryptography with structured photons in a turbulent free-space link ${ }^{11}$. However, harnessing high-dimensional spatial entanglement in the practical applications of large-scale quantum information processing still faces significant technological challenges. Conversely, entanglement in the energy-time domain is intrinsically suitable for long-distance transmission in fiber and free space ${ }^{12}$. Among the many manifestations of energy-time entanglement, photons carrying frequency entanglement have attracted great interest in recent years ${ }^{13-17}$. However, a concise yet efficient method for the creation and manipulation of arbitrary-dimensional frequency entanglement is still a formidable challenge. In addition, the characterization and verification of high-dimensional frequency entanglement also poses an ongoing challenge, owing to both the difficulty of performing required superposition measurements in the frequency domain, as well as the general challenges associated with performing full quantum state tomography in a large state space.

The objective of this work is two-fold: Firstly, we use spatial beating of Hong-Ou-Mandel (HOM) interference with polarizationfrequency hyperentangled photons on a beam splitter to discretize continuous and broadband spectra into a series of frequency bins. Since the temporal delay is set beyond the coherence time, it is equally likely to exhibit the bunching and anti-bunching effect, wherein only the anti-bunching term results in the correctly phased frequency-entangled photons. The bunched photon contribution is also problematic for the state analysis which is based on the second HOM interferometer, as they would result in a constant uncorrelated noise floor that would mask the detected two-photon beat signal due to frequency entanglement. As a direct result, the noisy bunched photons severely reduce the quality of quantum interference, which in turn will have negative impact on the efficiency of Bellstate measurement in quantum teleportation, the fidelity of the photonic quantum gates, the quality of entanglement swapping, etc. To tackle this issue, we exploit polarization anti-correlations of hyperentangled state to deterministically eliminate noise contributions that arise from detrimental photon bunching in the preparation stage, thus achieving high-fidelity frequency-bin entanglement. Secondly, we show how HOM interference can be used to characterize high-dimensional frequency entanglement; measurements of the fringe spacing of the observed interference pattern allow us to extract individual parameters even for high-dimensional entanglement. These results demonstrate that the modulation of temporal distinguishability in HOM interference provides a powerful tool for implementing highdimensional quantum information processing.

\footnotetext{
${ }^{1}$ Institute for Quantum Optics and Quantum Information - Vienna (IQOQI), Austrian Academy of Sciences, Boltzmanngasse 3, 1090 Vienna, Austria. ${ }^{2}$ Vienna Center for Quantum Science \& Technology (VCQ), Faculty of Physics, University of Vienna, Boltzmanngasse 5, 1090 Vienna, Austria. ${ }^{3}$ Department of Physics and Collaborative Innovation Center for Optoelectronic Semiconductors and Efficient Devices, Xiamen University, Xiamen 361005, China. ${ }^{4}$ Fraunhofer Institute for Applied Optics and Precision Engineering IOF, AlbertEinstein-Strasse 7, 07745 Jena, Germany. ${ }^{5}$ Friedrich Schiller University Jena, Abbe Center of Photonics, Albert-Einstein-Str. 6, 07745 Jena, Germany. ${ }^{凶}$ email: chenlx@xmu.edu.cn; Fabian.Steinlechner@iof.fraunhofer.de; Rupert.Ursin@oeaw.ac.at
} 

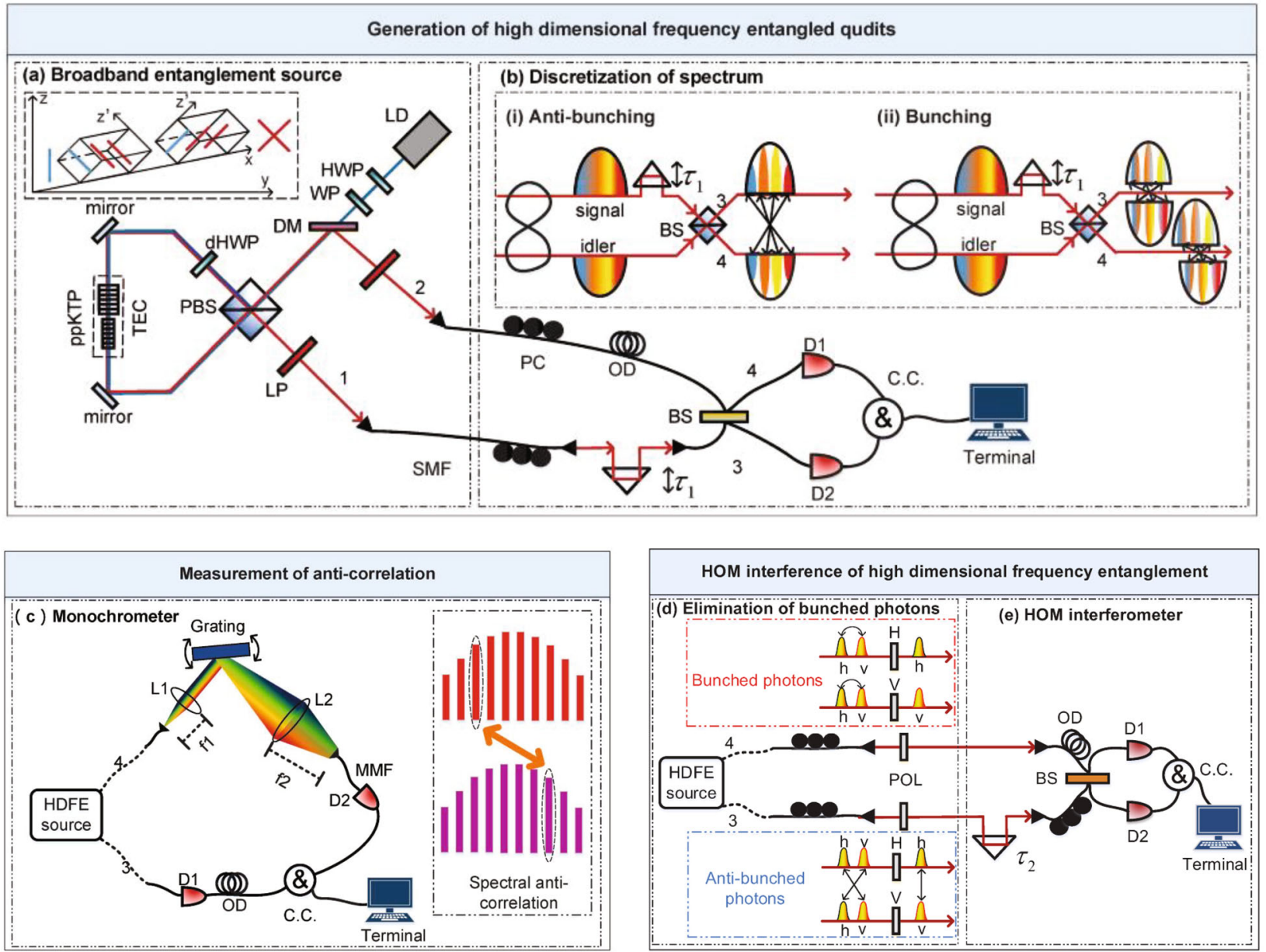

Fig. 1 Experimental setup. a Generation of high-dimensional frequency-entangled qudits through b Hong-Ou-Mandel interference. c Observation of frequency anti-correlations with a monochromator. Assisted by the elimination of spatially bunched photons d, HOM interference of high dimensional entanglement is observed e. LD laser diode, PBS polarizing beam splitter, HWP half wave plate, DM dichroic mirror, TEC temperature controller, LP long pass filter, SMF single-mode fiber, BS beam splitter, MMF multi-mode fiber, POL polarizer.

\section{RESULTS}

\section{Discretization of continuous frequency entanglement}

A major limiting factor for the number of entangled frequency modes, quantified by the dimensionality of entanglement, is the spectral bandwidth of photons. Type- 0 phase-matching yields the broadest spectral bandwidths among spontaneous parametric down-conversion (SPDC) processes ${ }^{18,19}$. As a direct result of the spatial overlap of the SPDC and pump modes, these type-0 entanglement sources requires wavelength distinguishability to route single photons into distinct spatial modes. However, this distinguishability limits their applicability in quantum information processing such as HOM interference. A question of the utmost importance and charm was raised: How can we prepare spatiallyseparated wavelength-degenerate polarization entanglement with high brightness. Recently, we present a polarization entanglement by using time-reversed HOM interference ${ }^{20}$ (see "Methods" for more details). By combining the benefits of Sagnactype sources ${ }^{21}$ and crossed-crystal sources ${ }^{22}$, wavelengthdegenerate polarization-entangled photons are deterministically routed into two distinct spatial modes (see Fig. 1a). Here the crystals are oriented at $45^{\circ}$ for the sake of phase compensation, thus the crystal are phase matched for SPDC with diagonally and anti-diagonally polarized pump beam. The resulting polarization-entangled state can be written in the form of
$|\psi\rangle=\frac{1}{\sqrt{2}}\left(\left|H_{1} V_{2}\right\rangle+\left|V_{1} H_{2}\right\rangle\right) \otimes\left|\omega_{s} \omega_{i}\right\rangle$, where $H(V)$ denotes horizontal (vertical) polarization, and $\omega_{s}$ and $\omega_{i}$ denote the continuous spectrum of signal and idler photons. Since the difference frequency $\Delta \omega=\left|\omega_{s}-\omega_{i}\right|$ of a color-entangled state typically exceeds that of the pump laser, continuous entanglement $|\Phi\rangle=$ $\frac{1}{\sqrt{2}}\left(\left|\omega_{s} \omega_{i}\right\rangle+\left|\omega_{i} \omega_{s}\right\rangle\right)$ arises quite naturally as a consequence of energy conservation.

Then, a HOM interferometer is used to project the continuous frequency spectrum into well-separated frequency bins without any requirement for spectrally selective filtering. As depicted in Fig. $1 \mathrm{~b}$, the photon in path 1 is then delayed relative to its partner photon in path 2 , introducing a relative time delay $\tau_{1}$ between the photons' time-of-arrival on a balanced beam splitter. For the incident frequency entanglement $|\Phi\rangle$, this optical delay would produce a phase shift that transforms the initial quantum state into $|\psi\rangle_{\text {hyper }}=\frac{1}{2}\left(\left|H_{1} V_{2}\right\rangle+\left|V_{1} H_{2}\right\rangle\right) \otimes\left(\left|\omega_{s} \omega_{i}\right\rangle+e^{i \Delta \omega \tau_{1}}\left|\omega_{i} \omega_{s}\right\rangle\right)$. This process comes to the HOM interference of photon pairs that are hyperentangled in two degrees of freedom, wherein the bunching and anti-bunching effects are related to the distinguishability in both of polarization and frequency domains ${ }^{23}$. Since the bunched photons are routed into the identical spatial modes and, conversely, the anti-bunched photons are routed into the opposite spatial modes, the bi-photon state after the beam 


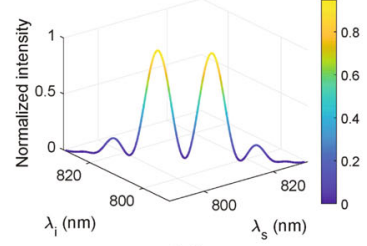

(a)

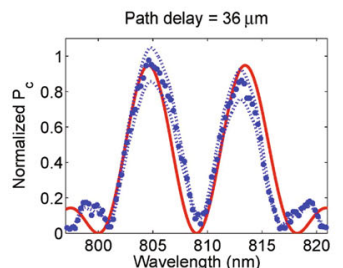

(e)

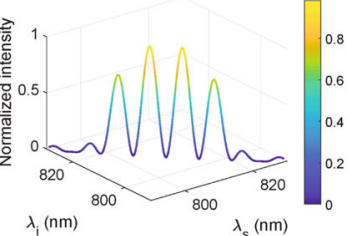

(b)

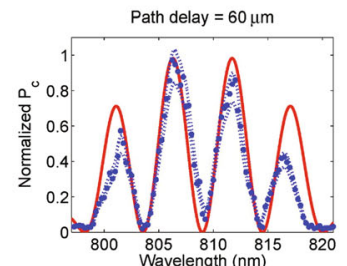

(f)

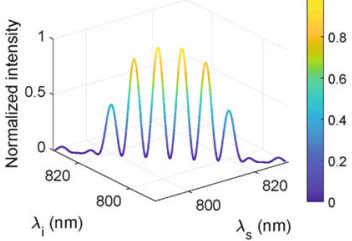

(c)

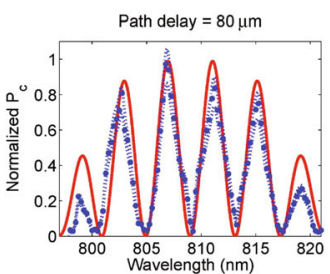

(g)

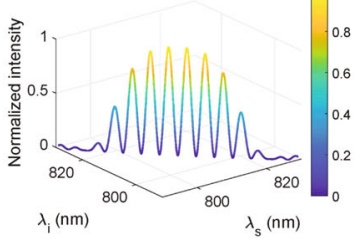

(d)

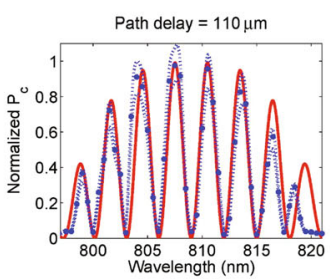

(h)

Fig. 2 Verification of high-dimensional frequency entanglement. a-d Theoretical simulation and e-h experimental observation of frequency anti-correlation by setting the relative path (time) delay at $\mathbf{a}$, e $36 \mu \mathrm{m}\left(\tau_{1}=0.12 \mathrm{ps}\right), \mathbf{b}, \mathbf{f} 60 \mu \mathrm{m}\left(\tau_{1}=0.2 \mathrm{ps}\right), \mathbf{c}, \mathbf{g} 80 \mu \mathrm{m}\left(\tau_{1}=0.27 \mathrm{ps}\right)$ and $\mathbf{d}, \mathbf{h} 110 \mu \mathrm{m}\left(\tau_{1}=0.37 \mathrm{ps}\right)$. The red lines represent the theoretical predictions, while the blue points represent the experimental results that are bounded by the standard deviation estimated by statistical methods assuming a Poisson distribution.

splitter is transformed to

$$
\begin{aligned}
|\psi\rangle_{\text {hyper }} \rightarrow & \frac{1}{2}\left[\left(\left|H_{3} V_{4}\right\rangle+\left|V_{3} H_{4}\right\rangle\right) \otimes|\psi\rangle_{\omega}^{-}\right. \\
& \left.+\left(\left|H_{3} V_{3}\right\rangle+\left|H_{4} V_{4}\right\rangle\right) \otimes|\psi\rangle_{\omega}^{+}\right],
\end{aligned}
$$

where $|\psi\rangle_{\omega}^{-}=\frac{1}{\sqrt{2}}\left(\left|\omega_{s} \omega_{i}\right\rangle-\left|\omega_{i} \omega_{s}\right\rangle\right) \quad\left(|\psi\rangle_{\omega}^{+}=\frac{1}{\sqrt{2}}\left(\left|\omega_{s} \omega_{i}\right\rangle+\left|\omega_{i} \omega_{s}\right\rangle\right)\right)$ denotes the frequency-entangled state produced in opposite (identical) spatial modes ${ }^{24}$. Thus, the frequency distribution of photon pairs that are separated in opposite spatial modes is discretized for filling the requirement of anti-bunching effect in the HOM interference. The drawback of this transformation is that the bi-photon component $|\psi\rangle_{\omega}^{+}$diminishes the interference visibility in the characterization process. To tackle this issue, we eliminate the detrimental bunched photon events via harnessing the anti-correlations of polarization-entangled state, e.g., the polarizer in spatial mode 3 is set at vertical polarization while the polarizer in spatial mode 4 is set at horizontal polarization (see Fig. $1 d$ ). This can be considered a state purification in the frequency domain and leads to a single non-vanishing term $\left|V_{3} H_{4}\right\rangle \otimes|\psi\rangle_{\omega}^{-}$. On the contrary, the contributions of bunched photons as $\left(\left|H_{3} V_{3}\right\rangle+\left|H_{4} V_{4}\right\rangle\right) \otimes|\psi\rangle_{\omega}^{+}$are completely erased. By identifying photons in opposite spatial modes, the normalized coincidence probability can be expressed as

$P\left(\tau_{1}\right)=\frac{1}{4} \iint d \omega_{1} d \omega_{2} f\left(\omega_{1}, \omega_{2}\right)\left|1-e^{i\left(\omega_{2}-\omega_{1}\right) \tau_{1}}\right|^{2}$.

This indicates that photon anti-bunching, i.e., coincidence events, only occur when bi-photons' frequency detuning satisfies $\left(\omega_{1}-\omega_{2}\right) \tau_{1}=(2 n+1) \pi$ ( $n$ is an integer). The intensity of bi-photon component in opposite spatial modes manifests itself as a sinusoidal oscillation within a Gaussian envelope, with an oscillation period related to $\tau_{1}$. As a consequence, arbitrarydimensional discrete frequency entanglement can be prepared by tuning the temporal distinguishability of frequency-entangled photons in HOM interference.

The joint spectral amplitude for different $\tau_{1}$ are plotted in Fig. 2 as a function of signal and idler wavelengths, wherein the experimental results almost agree well with theoretical prediction. The created discrete frequency-entangled state can be written as

$|\psi\rangle=\sum_{j=1}^{m / 2} A_{j}\left(a_{j}\left|\omega_{j} \omega_{m-j}\right\rangle-e^{i \varphi_{j}} a_{m-j}\left|\omega_{m-j} \omega_{j}\right\rangle\right)$,

where $m$ denotes the number of dimensions, $A_{j}$ is a probability amplitude, $\varphi_{j}$ is a phase-offset, $a_{j}^{2}=p_{j}, a_{j}^{2}+a_{m-j}^{2}=1, p_{j}$ is a
Table 1. Parameters extracted from Fig. 2.

\begin{tabular}{lllll}
\hline$\tau_{1}(\mathrm{ps})$ & $\Delta \lambda_{\mathrm{FWHM}}(\mathrm{nm})$ & $\mu_{j}(\mathrm{THz})$ & $p_{j}$ & $A_{j}$ \\
\hline 0.12 & 4.33 & 3.93 & 0.54 & - \\
0.20 & 2.83 & $6.94 / 2.67$ & $0.57 / 0.53$ & $0.36 / 0.65$ \\
0.27 & 2.11 & $5.71 / 1.94$ & $0.52 / 0.54$ & $0.46 / 0.54$ \\
0.37 & 1.52 & $6.91 / 4.09 / 1.42$ & $0.56 / 0.52 / 0.50$ & $0.24 / 0.35 / 0.41$ \\
\hline
\end{tabular}

$\Delta \lambda_{\text {FWHM }}$ is the single-photon frequency bandwidth, $\mu j=\left|\omega_{j}-\omega_{m-\mathrm{j}}\right|$ is the frequency detuning.

balance parameter and $\omega_{j}+\omega_{m-j}=\omega_{p}$. We note that the frequency bins are completely symmetric with respect to the central frequency. This agrees well with the fact that when wavelength-degenerate photons impinge on a beam splitter simultaneously, the coincidence probability falls to zero (so-called HOM dip) $)^{25}$, since all distinguishing information of the photons is erased.

\section{Experimental observation of frequency anti-correlation}

Polarization-entangled photon pairs are created in a Sagnac-type source (see Fig. 1a) at a degenerate wavelength of $810 \mathrm{~nm}$ with a spectral bandwidth of $\sim 20 \mathrm{~nm}$. They are produced at a pair rate of $160 \mathrm{kcps}$ per $\mathrm{mW}$ of pump power with a fidelity to the Polarization Bell state of $99.2 \%{ }^{20}$. The existence of multiple well-separated frequency bins is experimentally verified using a home-made single-photon monochromator (see Fig. 1c). By identifying twofold coincidences in opposite spatial modes 3 and 4 , we experimentally investigate anti-correlations of frequencyentangled qudits by setting the relative time delays at $0.12 \mathrm{ps}$, $0.2 \mathrm{ps}, 0.27 \mathrm{ps}$, and $0.37 \mathrm{ps}$. The experimental results are depicted in Fig. 2, which indicates a good separation of multiple frequency modes, and allows us to quantify the corresponding parameters as shown in Table 1.

\section{Characterization of high-dimensional frequency entanglement}

The characterization of frequency entanglement is challenging since the direct observation of interference visibilities in superposition bases of discrete frequency bins is a non-trivial task without nonlinear optics or time-resolved measurements ${ }^{26-29}$. 


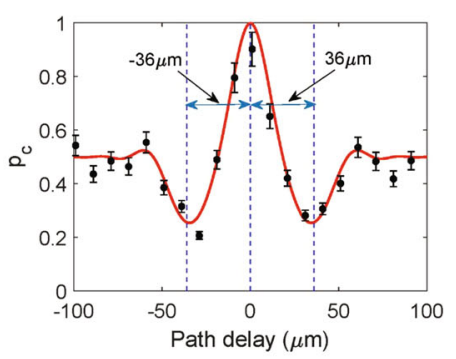

(a)

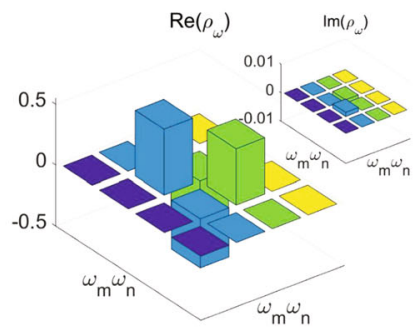

(d)

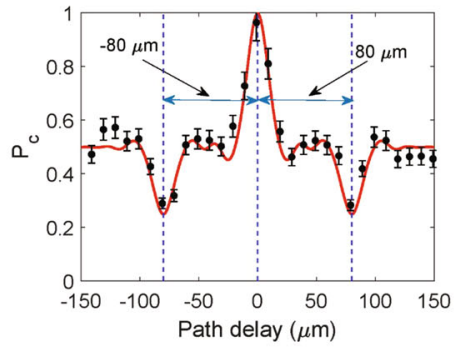

(b)

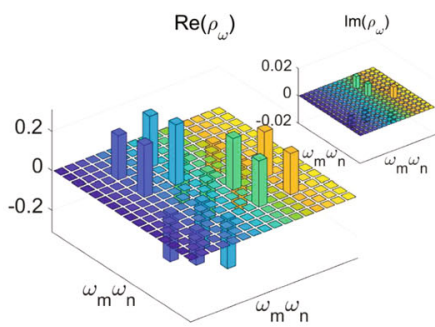

(e)

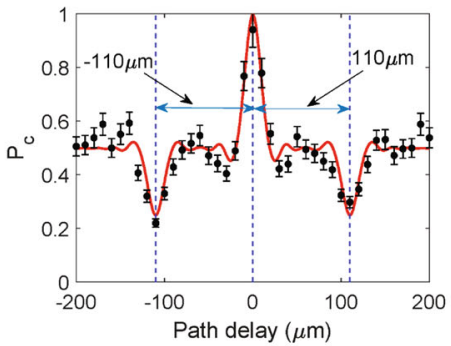

(c)

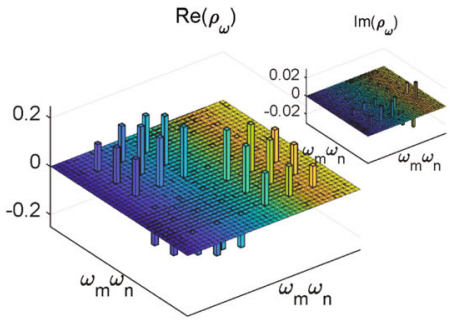

(f)

Fig. 3 Characterization of high-dimensional frequency entanglement. Spatial beating of discrete frequency-entangled photon pairs of $\mathbf{a}, \mathbf{d}$ two-dimensional frequency entanglement when setting $\tau_{1}=0.12 \mathrm{ps}(36 \mu \mathrm{m}), \mathbf{b}$, e four-dimensional frequency entanglement when setting $\tau_{1}=0.27 \mathrm{ps}(80 \mu \mathrm{m})$ and $\mathbf{c}$, $\mathbf{f}$ six-dimensional frequency entanglement when setting $\tau_{1}=0.37 \mathrm{ps}(110 \mu \mathrm{m})$. a-c Theoretical prediction and experimental results of the normalized coincidence rate, $\mathbf{d}-\mathbf{f}$ real and imaginary parts of the restricted density matrix. $\mathbf{d}$ The superposition states $\left|\omega_{m} \omega_{n}\right\rangle$ correspond to the basis of $\left|\omega_{1} \omega_{1}\right\rangle,\left|\omega_{1} \omega_{2}\right\rangle,\left|\omega_{2} \omega_{1}\right\rangle$ and $\left|\omega_{2} \omega_{2}\right\rangle$ in the two-dimensional space; e The superposition states $\left|\omega_{m} \omega_{n}\right\rangle$ correspond to the basis of $\left|\omega_{1} \omega_{n}\right\rangle,\left|\omega_{2} \omega_{n}\right\rangle,\left|\omega_{3} \omega_{n}\right\rangle$, and $\left|\omega_{4} \omega_{n}\right\rangle(n=1,2,3$, and 4) in the four-dimensional space; f The superposition states $\left|\omega_{m} \omega_{n}\right\rangle$ correspond to the basis of $\left|\omega_{1} \omega_{n}\right\rangle,\left|\omega_{2} \omega_{n}\right\rangle,\left|\omega_{3} \omega_{n}\right\rangle,\left|\omega_{4} \omega_{n}\right\rangle,\left|\omega_{5} \omega_{n}\right\rangle$, and $\left|\omega_{6} \omega_{n}\right\rangle(n=1,2,3,4,5$, and 6) in the six-dimensional space. The error bars are estimated by statistical methods assuming a Poisson distribution.

Here, we implement a non-local measurement of the coherences of a frequency-entangled state by spatial beating in HOM interference $^{30}$ (see Fig. 1d). The imbalance between two arms of a HOM interferometer introduces a relative time delay $\tau_{2}$, thus the coincidence probability $P\left(\tau_{1}, \tau_{2}\right)$ is obtained as

$$
\begin{aligned}
P\left(\tau_{1}, \tau_{2}\right)= & \frac{1}{16} \iint d \omega_{1} d \omega_{2} f\left(\omega_{1}, \omega_{2}\right) \mid e^{-i \omega_{2}\left(\tau_{1}+\tau_{2}\right)} \\
& +e^{-i\left(\omega_{1} \tau_{1}+\omega_{2} \tau_{2}\right)}+\left.e^{-i \omega_{1}\left(\tau_{1}+\tau_{2}\right)} e^{-i\left(\omega_{2} \tau_{1}+\omega_{1} \tau_{2}\right)}\right|^{2} .
\end{aligned}
$$

The parameter $\tau_{1}$ is set to be a constant that effectively determines the entanglement dimensionality, then $P\left(\tau_{1}, \tau_{2}\right)$ is simplified to the interference probability over the spatial beating delay $\tau_{2}$. Figure $3 a-c$ are the theoretical results of Eq. (4) by setting $\tau_{1}$ to be $0.12 \mathrm{ps}, 0.27 \mathrm{ps}$ and $0.37 \mathrm{ps}$, where two-, four- and sixdimensional frequency entanglement are prepared, respectively. The manifestation of interference fringes can be approximated by the sum of coincidence probabilities with different frequency detunings as ${ }^{31}$

$P\left(\tau_{2}\right)=\frac{1}{2}-\sum_{j=1}^{m / 2} \frac{V_{j}}{2} A_{j} \cos \left(\mu_{j} \tau_{2}+\varphi_{j}\right)\left(1-\left|\frac{2 \tau_{2}}{\tau_{c}}\right|\right)$,

for $\left|\tau_{2}\right| \leq \tau_{c} / 2$, where $V_{j}$ is the interference visibility quantifying the magnitude of oscillation, $\mu_{j}=\left|\omega_{j}-\omega_{m-j}\right|$ is frequency detuning of well-separated frequency-entangled bins, and $\tau_{c}$ denotes the single-photon coherence time that equals to the base-to-base envelope width. Curve fitting of experimental results to Eq. (5) reveal the corresponding parameters of a restricted density matrix in the high-dimensional state space.

\section{Experimental characterization of high-dimensional frequency entanglement}

We utilize two polarizers oriented at mutually orthogonal settings to eliminate the photon bunching components in Equation (1), as shown in the insets of Fig. $1 \mathrm{~d}$. In addition, polarization controllers are used to erase any polarization distinguishability. Then, the photons from each pair impinge on a balanced beam splitter from distinct input ports (see Fig. 1e). The non-classical spatial beating is revealed by scanning the arrival time of one of the photons incident on the beam splitter by $\tau_{2}$. The resulting interference fringes can be observed by identifying two-fold coincidence events between opposite output ports of the beam splitter. The experimental interference fringes for two-, four- and sixdimensional frequency entanglement created with delays $\tau_{1}=$ 0.12 ps, 0.27 ps, and 0.37 ps are depicted in Fig. 3a-c.

According to the quantum interference effect between twophoton probability amplitudes, the HOM interferometer has long been hailed as a versatile tool for analyzing the joint properties of photon pairs. Two-photon anti-bunching in the HOM interference is only possible if the photons are entangled in a specific state, anti-symmetric in the spatial modes ${ }^{32}$. Thus, observation of antibunching is an indication of entanglement in a degree of freedom, which might not be easily accessible in an experiment ${ }^{31}$. While the quantum interference reveals the joint properties of frequency entanglement, we state that the HOM interference cannot speak to the presence of high-dimensional frequency-bin entanglement in two-photon quantum frequency combs ${ }^{33}$. Since this method has been widely used in the verification of two-dimensional frequency entanglement ${ }^{34,35}$, we further extend it to the scenario of higher-dimensional state space. Let's first recall the coincidence probability of HOM interference of frequency-entangled photons as expressed in Eq. (5). It indicates that discrete frequencyentanglement manifests itself in sinusoidal oscillations of the interference fringes within a Gaussian envelope as a function of relative time delay, and the period of oscillation is determined by the frequency detuning of well-separated frequency-entangled bins. As the coherence time of frequency-entangled photons is determined by the single-photon frequency bandwidth (related to the first time delay $\tau_{1}$ ), the various periods of oscillation are sufficient for us to extract the corresponding parameters by using 
Table 2. Parameters extracted from Fig. 3.

\begin{tabular}{lllllll}
\hline$\tau_{1}(\mathrm{ps})$ & $\tau_{c}(\mathrm{ps})$ & $\Delta \lambda_{\mathrm{FWHM}}(\mathrm{nm})$ & $\mu_{j}(\mathrm{THz})$ & $A_{j}$ & $V_{j}$ & $\varphi_{j}\left(^{\circ}\right)$ \\
\hline 0.12 & 0.47 & 4.24 & 4.01 & - & 0.81 & 179.83 \\
0.27 & 0.94 & 2.13 & $5.71 / 1.94$ & $0.44 / 0.56$ & $0.80 / 0.86$ & $180.03 / 182.14$ \\
0.37 & 1.43 & 1.42 & $6.54 / 4.07 / 1.48$ & $0.20 / 0.38 / 0.42$ & $0.80 / 0.94 / 0.93$ & $172.48 / 181.23 / 177.67$ \\
\hline
\end{tabular}

nonlinear curve-fitting. For an instructive means of understanding, this approach can be considered as a quantum version of spectrum analysis, which decomposes the complex periodic vibration into a series of simple harmonic motion. Table 2 shows the corresponding parameters that is revealed by a fit of our experimental measurement data as shown in Fig. 3a-c to Eq. (5). Backed by the observation of frequency anti-correlations and HOM interference in high-dimensional space, we confirm that the produced state is actually close to the form of Eq. (3) (see the next section for more details). While these experimental measurements are insufficient for full tomography of high-dimensional entanglement, they reveal the parameters of the restricted density matrices ${ }^{31}$, which are shown in Fig. 3d-f. Thereinto, several density-matrix elements are set to zero, because of energy conservation in the process of SPDC with a narrow-band pump laser. We note that our experimental results agree well with theoretical prediction, while slight deviations can be attributed to misalignment and imperfect optical components.

On the basis of the previous discussion, the HOM interference dip appears when $\tau_{2}$ is odd times of $\tau_{1}$, while peak appears when $\tau_{2}$ is even times of $\tau_{1}$. Additionally, since the spectrum manifests itself as a characteristic sinusoidal function, the single-photon frequency bandwidth can be approximated as $\Delta f_{\mathrm{FWHM}} \approx \frac{\mu_{1}^{\lambda} \mathrm{c}}{2 \lambda^{2}}$, corresponding to coherence time $\tau_{c}=\frac{0.885}{\Delta f_{\mathrm{FWHM}}} \sim 3.54 \tau_{1}{ }^{34}$. Thus, limited by the coherence time, we are able to observe interference dips at $\tau_{2}= \pm \tau_{1}$ and an interference peak at $\tau_{2}=0$.

Our strategy allows one to learn the entanglement structure by spectral analysis of the HOM interference pattern formed by spatial beating of frequency entangled photon pairs. Implementation simplicity, reduced number of measurements, and accurate estimation could make this approach practical and convenient for the characterization of high-dimensional frequency entanglement.

\section{Dimensionality estimation of high-dimensional frequency entanglement}

Entanglement dimensionality is an important metric, which reveals the minimum number of dimensions that is needed to faithfully reproduce correlations of the state in any global product basis. Based on the observation of frequency anti-correlations and coherent superpositions in two-dimensional subspaces $\left\{\left|\omega_{j}\right\rangle,\left|\omega_{m-j}\right\rangle\right\}$, the produced state is actually close to the form of Eq. (3). The measurements performed serve as testbeds for our frequency-bin entangled source. For using these states in a quantum communication setting with local certification of entanglement, two further ingredients will be needed. In particular, we will need local superposition measurements of different frequency modes $27,29,36$ to enable spatially-separated quantum communication protocols and we will need to ascertain the visibilities in all pairs of frequency subspaces. Nonetheless, our experiment serves as a source characterisation, showing the clear potential of the source and the notion of frequency modes for generating high-dimensional entanglement. In fact, assuming that the visibilites in all $m(m-1) / 2$ frequency subspaces correspond to the average visibility of the measured two-dimensional subspaces, the achievable entanglement of formation in our experiment is $E_{2}=0.57, E_{4}=1.05$ and $E_{6}=1.56$ for the two-, four- and six-dimensional frequency modes ${ }^{37}$.

\section{DISCUSSION}

We have presented a method of exploiting temporal distinguishability in HOM interference to generate, characterize, and verify high-dimensional entanglement in frequency-bin qudits. In analogy to double-slit interference, the manipulation of temporal distinguishability in HOM interference of entangled photons is sufficient to prepare arbitrary-dimensional discrete frequency entanglement. With the assistance of hyperentanglement, it is allowed to eliminate the detrimental bunching photons by using polarization anti-correlation, and thus make the scheme compelling for the applications with the requirement of post-detectionfree. Another remarkable trait of this work is that flexible control of temporal distinguishability in HOM interference is sufficient to characterize the generated high-dimensional frequency entanglement. It is an alternative route to perform quantum state tomography in frequency degree of freedom, and thus avoid the need of complex nonlinear optics or expensive time-resolved detectors. We believe this scheme paves an alternative route towards harnessing high-dimensional entanglement, and maybe a good candidate for next generation of quantum information processing.

HOM interferometers incorporating unbalanced beam splitters may enable the tailoring of frequency-entangled photons with even richer structures. This approach may also provide valuable possibilities of engineering more complex forms of quantum states such as high-dimensional asymmetric states ${ }^{38}$. In addition, we show that the finally observed interference can be considered as the sum of HOM interference of frequency sub-entanglement in two-dimensional Hilbert space. Therefore, this approach is a powerful tool for revealing relevant quantum information from single interference pattern, which elevates the performance of HOM interference in the applications of simultaneous quantum measurement. We hope that our results inspire not only practical quantum communication and quantum computation applications based on high-dimensional frequency entanglement, but also more experimental configurations exploiting quantum interference in other degrees of freedom, e.g., orbital angular momentum ${ }^{39}$.

Our approach reveals a connection between spectral and temporal degrees of freedom, which may have potential applications in quantum optical coherence tomography and quantum spectral analysis. For example, spectral-domain optical coherence tomography (SD-OCT) is a non-invasive diagnostic technique that can extract the depth information of an optically transparent or translucent sample from spectral analysis ${ }^{40-42}$. Since our approach indicates that the two-photon joint spectral intensity is associated to the relative time delay, it can be extended to implement a quantum version of SD-OCT, which is an essential requisite for measuring photon-sensitive biological and chemical samples. Additionally ${ }^{43}$, states that difference-frequency distribution of biphoton wavefunctions can be obtained by making a Fourier transform on the HOM interference pattern. Backed by our results, it is possible to construct an entanglementassisted quantum interferometric spectrometer based on HOM interferometry. Although this work only reports the advantages of our approach in harnessing high-dimensional frequency entanglement, similar great enhancement can also be achieved for a variety of applications like quantum metrology that is used to 
detect tiny displacement or phase shift. We would like to leave these possibilities of applications in our further experimental studies in the future.

\section{METHODS}

\section{High-dimensional frequency entanglement source}

In our experimental realization of ultra-bright entanglement source, two mutually orthogonally oriented $11.48-\mathrm{mm}$-long ppKTP crystals are manufactured to provide collinear phase matching with pump (p), signal (s) and idler (i) photons at center wavelengths of $\lambda_{p} \approx 405 \mathrm{~nm}$ and $\lambda_{s, i} \approx$ $810 \mathrm{~nm}$. They are pumped with a $405 \mathrm{~nm}$ continuous wave gratingstabilized laser diode. The horizontally polarized pump light, propagates in clockwise direction, can generate a pair of photons in a state as

$|\psi\rangle_{C}=\frac{\left|2_{D}, 0_{A}\right\rangle+e^{i \phi}\left|0_{D}, 2_{A}\right\rangle}{\sqrt{2}}$,

where $\left|2_{D}, 0_{A}\right\rangle$ and $\left|0_{D}, 2_{A}\right\rangle$ represent the superposition state corresponding to both of two photons are diagonally or anti-diagonally polarized, $\phi$ represents a polarization dependent phase factor. By setting the relative phase $\phi=\pi$, this states becomes

$$
|\psi\rangle_{C}=\frac{\left|2_{D}, 0_{A}\right\rangle-\left|0_{D}, 2_{A}\right\rangle}{\sqrt{2}}=\left|1_{H}, 1_{V}\right\rangle_{C}
$$

which can be seen as the time-reversed HOM interference. In analogy, the vertically polarized pump light, propagates in counter-clockwise direction, can generate a pair of photons in a state as $|\psi\rangle_{a}=\left|1_{H}, 1_{\mathrm{V}}\right\rangle_{a}$. Thus, the coherent superposition of pair-generation possibilities $|\psi\rangle_{c}$ and $|\psi\rangle_{a}$ would result in the state in the form of $|\psi\rangle=\frac{1}{\sqrt{2}}\left(\left|H_{1} V_{2}\right\rangle+\left|V_{1} H_{2}\right\rangle\right) \otimes\left|\omega_{s} \omega_{i}\right\rangle$.

The arriving time of one of pair of photons at the beam splitter is scanned to accomplish the task of observation of HOM interference. Polarization controllers are required to compensate the phase drift caused by fluctuation and imperfect components. Finally, the anti-bunched photons are detected by silicon avalanche photon diodes, and two-fold events are identified using a fast electronic AND gate when two photons arrive at the detectors within a coincidence window of $\sim 3 \mathrm{~ns}$.

\section{HOM interference of high-dimensional frequency entanglement}

The typical form of entangled state generated in SPDC process can be described as

$$
|\psi\rangle=\int_{0}^{\infty} \int_{0}^{\infty} d \omega_{s} d \omega_{i} f\left(\omega_{s}, \omega_{i}\right) \hat{a}_{s}^{\dagger}\left(\omega_{s}\right) \hat{a}_{i}^{\dagger}\left(\omega_{i}\right)|0\rangle,
$$

To investigate the frequency dependent phase, we introduce a tunable time delay $\tau_{1}$, and it generates a phase shift $\exp \left(-i \omega_{i} \tau_{1}\right)$ to the idler photon. Then we can engineer high dimensional frequency entangled qudits by applying the operations of beam splitter to frequency entanglement as follows

$$
\begin{aligned}
& \hat{a}_{s}^{\dagger}\left(\omega_{s}\right)=\frac{1}{\sqrt{2}}\left[i \hat{a}_{1}^{\dagger}\left(\omega_{s}\right)+\hat{a}_{2}^{\dagger}\left(\omega_{s}\right)\right] \\
& \hat{a}_{i}^{\dagger}\left(\omega_{i}\right)=\frac{1}{\sqrt{2}}\left[\hat{a}_{1}^{\dagger}\left(\omega_{i}\right)+i \hat{a}_{2}^{\dagger}\left(\omega_{i}\right)\right] .
\end{aligned}
$$

After performing the operation of HOM interferometer, we obtain the two-photon state as

$$
\begin{aligned}
|\psi\rangle= & \frac{1}{2} \int_{0}^{\infty} \int_{0}^{\infty} d \omega_{s} d \omega_{i} f\left(\omega_{s}, \omega_{i}\right) e^{-i \omega_{i} \tau_{1}}\left[i \hat{a}_{1}^{\dagger}\left(\omega_{s}\right) \hat{a}_{1}^{\dagger}\left(\omega_{i}\right)\right. \\
& \left.+i \hat{a}_{2}^{\dagger}\left(\omega_{s}\right) \hat{a}_{2}^{\dagger}\left(\omega_{i}\right)+\hat{a}_{1}^{\dagger}\left(\omega_{i}\right) \hat{a}_{2}^{\dagger}\left(\omega_{s}\right)-\hat{a}_{1}^{\dagger}\left(\omega_{s}\right) \hat{a}_{2}^{\dagger}\left(\omega_{i}\right)\right]|0\rangle,
\end{aligned}
$$

where subscript $1 / 2$ represent two output modes of beam splitter. For the post-selected coincidence counts by two detectors, only last two terms of Eq. (10) can remain. As two individual photons after beam splitter are undistinguishable, we substitute $\omega_{s}$ and $\omega_{i}$ with $\omega_{1}$ and $\omega_{2}$. By applying $e^{-i \omega_{i} \tau_{1}}$ for cancelling global phase, we obtain

$$
\begin{aligned}
|\psi\rangle= & \frac{1}{2} \int_{0}^{\infty} \int_{0}^{\infty} d \omega_{1} d \omega_{2}\left[f\left(\omega_{1}, \omega_{2}\right)\right. \\
& \left.-f\left(\omega_{2}, \omega_{1}\right) e^{-i\left(\omega_{1}-\omega_{2}\right) \tau_{1}}\right] \hat{a}_{1}^{\dagger}\left(\omega_{1}\right) \hat{a}_{2}^{\dagger}\left(\omega_{2}\right)|0\rangle .
\end{aligned}
$$

Finally, the coincidence probability $P\left(\tau_{1}\right)$ as a function of time delay can be expressed as Eq. (2). Next we consider the interference in the second HOM interferometer, where the incident quantum state can be expressed as

$|\psi\rangle=\int_{0}^{\infty} \int_{0}^{\infty} d \omega_{s} d \omega_{i} g\left(\omega_{s}, \omega_{i}\right) \hat{a}_{s}^{\dagger}\left(\omega_{s}\right) \hat{a}_{i}^{\dagger}\left(\omega_{i}\right)|0\rangle$,

where $g\left(\omega_{1}, \omega_{2}\right)=f\left(\omega_{1}, \omega_{2}\right) e^{-i \omega_{2} \tau_{1}}-f\left(\omega_{2}, \omega_{1}\right) e^{-i \omega_{1} \tau_{1}}$. Analogously, the coincidence probability $P\left(\tau_{2}\right)$ as a function of time delay can be expressed as:

$P\left(\tau_{2}\right)=\frac{1}{4} \int_{0}^{\infty} \int_{0}^{\infty} d \omega_{1} d \omega_{2}\left|g\left(\omega_{1}, \omega_{2}\right)-g\left(\omega_{2}, \omega_{1}\right) e^{-i\left(\omega_{1}-\omega_{2}\right) \tau_{2}}\right|^{2}$

By replacing $g\left(\omega_{1}, \omega_{2}\right)$ with joint spectral amplitude from SPDC source, we could obtain coincidence probability as a function of two time delays as Eq. (4).

\section{DATA AVAILABILITY}

Data are available from the authors on reasonable request.

Received: 26 January 2021; Accepted: 20 October 2021; Published online: 07 December 2021

\section{REFERENCES}

1. Ecker, S. et al. Overcoming noise in entanglement distribution. Phys. Rev. $X \mathbf{9}$, 041042 (2019).

2. Dada, A. C., Leach, J., Buller, G. S., Padgett, M. J. \& Andersson, E. Experimental high-dimensional two-photon entanglement and violations of generalized bell inequalities. Nat. Phys. 7, 677 (2011).

3. Mirhosseini, M. et al. High-dimensional quantum cryptography with twisted light. New J. Phys. 17, 033033 (2015).

4. Raussendorf, R. \& Harrington, J. Fault-tolerant quantum computation with high threshold in two dimensions. Phys. Rev. Lett. 98, 190504 (2007).

5. Molina-Terriza, G., Torres, J. P. \& Torner, L. Twisted photons. Nat. Phys. 3, 305 (2007).

6. Zhong, T. et al. Photon-efficient quantum key distribution using time-energy entanglement with high-dimensional encoding. New J. Phys. 17, 022002 (2015).

7. Fickler, R. et al. Interface between path and orbital angular momentum entanglement for high-dimensional photonic quantum information. Nat. Commun. 5, 4502 (2014).

8. Kovlakov, E., Bobrov, I., Straupe, S. \& Kulik, S. Spatial bell-state generation without transverse mode subspace postselection. Phys. Rev. Lett. 118, 030503 (2017).

9. Bozinovic, N. et al. Terabit-scale orbital angular momentum mode division multiplexing in fibers. Science 340, 1545-1548 (2013).

10. Gregg, P., Kristensen, P. \& Ramachandran, S. Conservation of orbital angular momentum in air-core optical fibers. Optica 2, 267-270 (2015).

11. Sit, A. et al. High-dimensional intracity quantum cryptography with structured photons. Optica 4, 1006-1010 (2017).

12. Steinlechner, F. et al. Distribution of high-dimensional entanglement via an intracity free-space link. Nat. Commun. 8, 15971 (2017).

13. Kuzucu, O., Fiorentino, M., Albota, M. A., Wong, F. N. \& Kärtner, F. X. Two-photon coincident-frequency entanglement via extended phase matching. Phys. Rev. Lett. 94, 083601 (2005).

14. Roslund, J., De Araujo, R. M., Jiang, S., Fabre, C. \& Treps, N. Wavelengthmultiplexed quantum networks with ultrafast frequency combs. Nat. Photonics $\mathbf{8}$, 109 (2014).

15. Yan, H. et al. Generation of narrow-band hyperentangled nondegenerate paired photons. Phys. Rev. Lett. 106, 033601 (2011).

16. Lingaraju, N. B. et al. Quantum frequency combs and hong-ou-mandel interferometry: the role of spectral phase coherence. Opt. Express 27, 38683-38697 (2019).

17. Orre, V. V. et al. Interference of temporally distinguishable photons using frequency-resolved detection. Phys. Rev. Lett. 123, 123603 (2019).

18. Wengerowsky, S. et al. An entanglement-based wavelength-multiplexed quantum communication network. Nature 564, 225-228 (2018).

19. Steinlechner, F. et al. A high-brightness source of polarization-entangled photons optimized for applications in free space. Opt. Express 20, 9640-9649 (2012).

20. Chen, Y. et al. Polarization entanglement by time-reversed hong-ou-mandel interference. Phys. Rev. Lett. 121, 200502 (2018).

21. Fedrizzi, A., Herbst, T., Poppe, A., Jennewein, T. \& Zeilinger, A. A wavelengthtunable fiber-coupled source of narrowband entangled photons. Opt. Express 15, 15377-15386 (2007). 
22. Kwiat, P. G., Waks, E., White, A. G., Appelbaum, I. \& Eberhard, P. H. Ultrabright source of polarization-entangled photons. Phys. Rev. A 60, R773-R776 (1999).

23. Wang, X.-L. et al. Quantum teleportation of multiple degrees of freedom of a single photon. Nature 518, 516-519 (2015).

24. Jin, R.-B. et al. Simple method of generating and distributing frequencyentangled qudits. Quantum Sci. Technol. 1, 015004 (2016).

25. Hong, C. K., Ou, Z. Y. \& Mandel, L. Measurement of subpicosecond time intervals between two photons by interference. Phys. Rev. Lett. 59, 2044-2046 (1987).

26. Kues, M. et al. On-chip generation of high-dimensional entangled quantum states and their coherent control. Nature 546, 622 (2017).

27. Lu, H.-H. et al. Electro-optic frequency beam splitters and tritters for highfidelity photonic quantum information processing. Phys. Rev. Lett. 120, 030502 (2018).

28. Maclean, J. P. W., Donohue, J. M. \& Resch, K. J. Direct characterization of ultrafast energy-time entangled photon pairs. Phys. Rev. Lett. 120, 53601 (2018)

29. Brecht, B., Reddy, D. V., Silberhorn, C. \& Raymer, M. G. Photon temporal modes: a complete framework for quantum information science. Phys. Rev. $X$ 5, 1-17 (2015).

30. Ou, Z. Y. \& Mandel, L. Observation of spatial quantum beating with separated photodetectors. Phys. Rev. Lett. 61, 54-57 (1988).

31. Fedrizzi, A. et al. Anti-symmetrization reveals hidden entanglement. New J. Phys. 11, 103052 (2009).

32. Mattle, K., Weinfurter, H., Kwiat, P. G. \& Zeilinger, A. Dense coding in experimenta quantum communication. Phys. Rev. Lett. 76, 4656-4659 (1996).

33. Lingaraju, N. B. et al. Quantum frequency combs and hong-ou-mandel interferometry: the role of spectral phase coherence. Opt. Express 27, 38683-38697 (2019).

34. Ramelow, S., Ratschbacher, L., Fedrizzi, A., Langford, N. \& Zeilinger, A. Discrete tunable color entanglement. Phys. Rev. Lett. 103, 253601 (2009).

35. Chen, Y. et al. Verification of high-dimensional entanglement generated in quantum interference. Phys. Rev. A 101, 032302 (2020).

36. Lu, H.-H., Simmerman, E. M., Lougovski, P., Weiner, A. M. \& Lukens, J. M. Fully arbitrary control of frequency-bin qubits. Phys. Rev. Lett. 125, 120503 (2020).

37. Martin, A. et al. Quantifying photonic high-dimensional entanglement. Phys. Rev. Lett. 118, 110501 (2017).

38. Erhard, M., Malik, M., Krenn, M. \& Zeilinger, A. Experimental greenberger-hornezeilinger entanglement beyond qubits. Nat. Photonics 12, 759-764 (2018).

39. Zhou, H.-L. et al. Orbital angular momentum complex spectrum analyzer for vortex light based on the rotational doppler effect. Light Sci. Appl. 6, e16251-e16251 (2017)

40. Kalkman, J., Sprik, R. \& van Leeuwen, T. G. Path-length-resolved diffusive particle dynamics in spectral-domain optical coherence tomography. Phys. Rev. Lett. 105, 198302 (2010).

41. Marchand, P. J., Riemensberger, J., Skehan, J. C., Ho, J. J. \& Kippenberg, T. J. Soliton microcomb based spectral domain optical coherence tomography. Nat. Commun. 12, 427 (2021).

42. Siddiqui, M. et al. High-speed optical coherence tomography by circular interferometric ranging. Nat. Photonics 12, 111-116 (2018).

43. Jin, R.-B. \& Shimizu, R. Extended wiener-khinchin theorem for quantum spectral analysis. Optica 5, 93-98 (2018).

\section{ACKNOWLEDGEMENTS}

Financial support from the Austrian Research Promotion Agency (FFG) Projects Agentur für Luft- und Raumfahrt (FFG-ALR contract 6238191 and 866025), the European Space Agency (ESA contract 4000112591/14/NL/US) as well as the Austrian Academy of Sciences is gratefully acknowledged. F.S. acknowledges financial support from Fraunhofer Internal Programs under Grant No. Attract 066-604178. Y.C. and L.C. acknowledge financial support from the National Natural Science Foundation of China $(12034016,12004318,61975169)$, the Fundamental Research Funds for the Central Universities at Xiamen University (20720190057), the Natural Science Foundation of Fujian Province of China (2020J05004), the Fundamental Research Funds for the Central Universities at XMU (20720200074).

\section{AUTHOR CONTRIBUTIONS}

Y.C. developed the initial idea for this work. Y.C. conducted the experiment under supervision from L.C., F.S., and R.U. with the help of S.E. and M.H. All authors contributed to the final version of the manuscript.

\section{COMPETING INTERESTS}

The authors declare no competing interests.

\section{ADDITIONAL INFORMATION}

Correspondence and requests for materials should be addressed to Lixiang Chen, Fabian Steinlechner or Rupert Ursin.

Reprints and permission information is available at http://www.nature.com/ reprints

Publisher's note Springer Nature remains neutral with regard to jurisdictional claims in published maps and institutional affiliations.

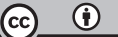

Open Access This article is licensed under a Creative Commons Attribution 4.0 International License, which permits use, sharing, adaptation, distribution and reproduction in any medium or format, as long as you give appropriate credit to the original author(s) and the source, provide a link to the Creative Commons license, and indicate if changes were made. The images or other third party material in this article are included in the article's Creative Commons license, unless indicated otherwise in a credit line to the material. If material is not included in the article's Creative Commons license and your intended use is not permitted by statutory regulation or exceeds the permitted use, you will need to obtain permission directly from the copyright holder. To view a copy of this license, visit http://creativecommons. org/licenses/by/4.0/.

(c) The Author(s) 2021 